\title{
Distinct Triglyceride-Glucose Trajectories are associated with Different Risks of Incident Cardiovascular Disease in Normal- Weight Adults
}

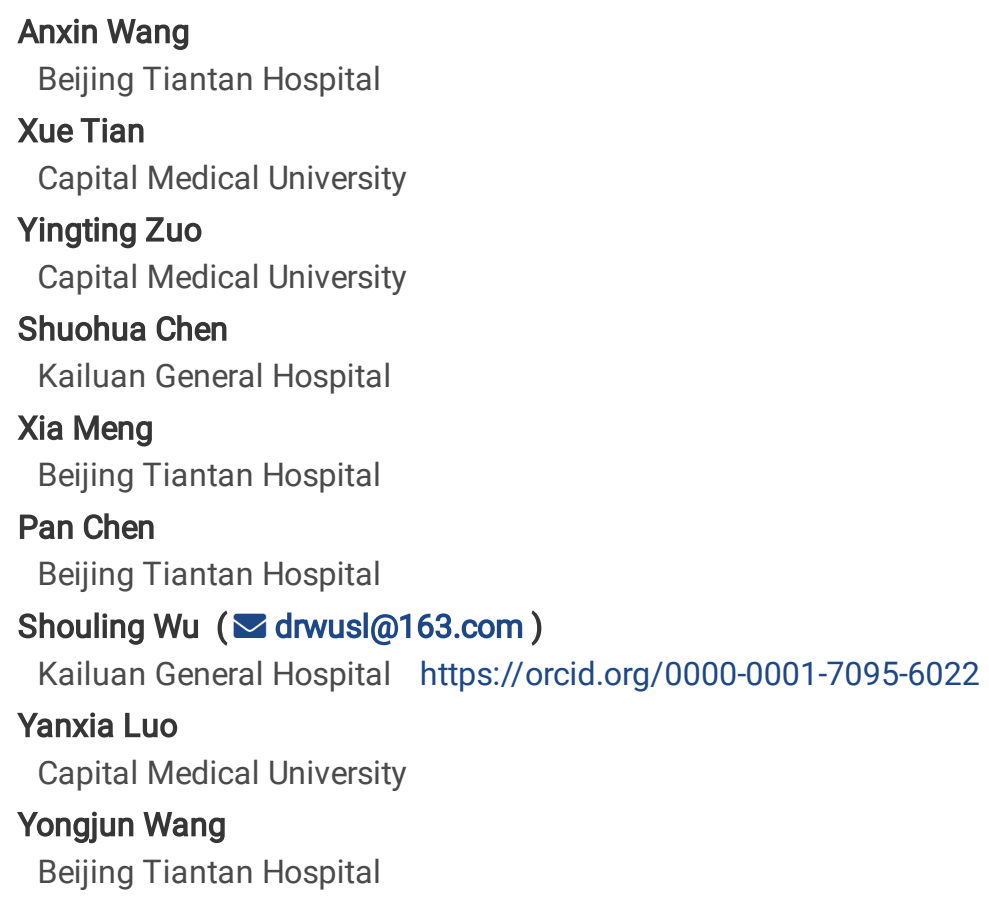

Anxin Wang

Beijing Tiantan Hospital

Xue Tian

Capital Medical University

Yingting Zuo

Capital Medical University

Shuohua Chen

Kailuan General Hospital

Xia Meng

Beijing Tiantan Hospital

Pan Chen

Beijing Tiantan Hospital

Shouling Wu ( $\nabla$ drwusl@163.com )

Kailuan General Hospital https://orcid.org/0000-0001-7095-6022

Yanxia Luo

Capital Medical University

Yongjun Wang

Beijing Tiantan Hospital

Original investigation

Keywords: Triglyceride-glucose index, Trajectories analysis, cardiovascular disease, normal weight

Posted Date: June 30th, 2021

DOI: https://doi.org/10.21203/rs.3.rs-649230/v1

License: @ (1) This work is licensed under a Creative Commons Attribution 4.0 International License. Read Full License 


\section{Abstract}

\section{Background}

Risk of cardiovascular disease (CVD) is increased in metabolically obese normal weight adults. However, we have limited knowledge of how to prevent CVD in normal-weight people. The triglyceride-glucose index (TyG index) has been considered as a contributor of CVD, while long-term patterns of the triglyceride-glucose index (TyG index) and their effects on CVD among normal-weight adults are poorly characterized. Therefore, this study aimed to identify TyG index trajectories in normal-weight adults and to determine their association with the risk of incident CVD.

\section{Methods}

This prospective cohort study included 40,473 normal-weight participants who were free of stroke and myocardial infarction prior to or in 2012. The TyG index was calculated as In [fasting triglyceride ( $\mathrm{mg} / \mathrm{dL}) \times$ fasting glucose $(\mathrm{mg} / \mathrm{dL}) / 2$ ], and the TyG index trajectories during 2006-2012 were identified by latent mixture modeling. Cox proportional hazards models were used to examine the associations between TyG index trajectories and incident CVD.

\section{Results}

We identified five distinct TyG index trajectories according to TyG index range and changing pattern over time: low-stable ( $n=9,806$; mean TyG index 7.84-7.93), moderate-stable ( $n=22,066$; mean TyG index 8.43-8.52), elevated-decreasing $(n=1,469 ;$ mean TyG index 9.838.75), moderate-increasing ( $n=5,842$; mean TyG index 8.98-9.26), and elevated-stable ( $n=1,290$; mean TyG index 9.91-10.07). During 6.74 years of follow-up, we documented 1,577 incident CVD events. Compared with the low-stable pattern, the highest risk of CVD was observed in the elevated-stable pattern (hazard ratio [HR], 2.24; 95\% confidence interval [Cl]: 1.73-2.90), followed by the moderateincreasing pattern ( $\mathrm{HR}, 1.70 ; 95 \% \mathrm{Cl}, 1.43-2.04)$, the elevated-decreasing pattern ( $\mathrm{HR}, 1.45 ; 95 \% \mathrm{Cl}, 1.11-1.89)$, and the moderate-stable pattern (HR, 1.25; $95 \% \mathrm{Cl}, 1.08-1.44)$. Similar results were also observed for stroke and myocardial infarction. Consistently, annual increment of the TyG index during 2006-2012 also predicted future risk of CVD.

\section{Conclusions}

Distinct TyG index trajectories were significantly associated differently subsequent risk of CVD in normal-weight individuals. These observations suggested that long-term trajectories of TyG index may be useful for predicting CVD among normal-weight adults.

\section{Introduction}

Insulin resistance (IR) is either a precursor or a pivotal component of numerous chronic diseases[1-5] including cardiovascular disease (CVD)[6], which is the leading cause of mortality and accounts for more than $40 \%$ of deaths in China.[7] The triglyceride-glucose index (TyG index), a product of fasting triglyceride (TG) and fasting blood glucose (FBG), has been evaluated as a reliable, simple and inexpensive surrogate for IR, and has high correlation with hyperinsulinaemic-euglycaemic clamp (the gold standard technique for assessing IR).[8-10] Numerous studies have found that increasing TyG index level was an independent risk factor for incident CVD.[1115] It is worth noting that the majority of published studies on this topic were based on a single measurement of the TyG index, failing to take into account the potential effects of heterogeneous long-term patterns in TyG index, which could bias the true relation between the TyG index and CVD toward the null hypothesis. Furthermore, although CVD presents suddenly, the advanced extensive complex intramural lesions that lead to plaque rupture develop over decades.[16] Thus, prospective studies that evaluate the effects of long-term TyG index trajectory patterns on CVD are essential.

Concurrently, up to $30 \%$ of normal weight individuals are characterized by a cluster of cardiometabolic risk factors that are typically only seen in obese individuals, the so called "metabolically obese normal weight (MONW)", and individuals with MONW have a higher susceptibility to CVD.[17-19] Moreover, normal-weight people may not monitor their health indicators or take prevention measures for CVD. Therefore, early identification of CVD for normal-weight people is necessary. Particularly Chinese people, despite their lower absolute body mass index (BMI), are more prone to IR than Western populations.[20, 21] Nevertheless, data are limited on the prevention of CVD for normal-weight people.

Therefore, in the present study, we aimed to identify distinct trajectories of TyG index in normal-weight participants who share similar trajectories in TyG index during a 6-year exposure period and analyzed their relationships with future risk of CVD. 


\section{Methods}

\section{Study population}

Data were obtained from the Kailuan study, which is prospective cohort study conducted in the Kailuan community in Tangshan, China. Detailed study design and procedures have been described previously.[22-24] Briefly, since June 2006, a total of 101,510 participants (81,110 men and 20,400 women, aged 18-98 years) were enrolled in the baseline survey and completed questionnaires, health assessments and laboratory tests every 2 years. In the present study, we examined data for 49,749 participants with normal weight (BMI $18.5-24.9 \mathrm{~kg} / \mathrm{m}^{2}$ ) according to the Guidelines and Prevention and Control of Overweight and Obesity in Chinese Adults.[25] The TyG trajectories were developed from 2006 to 2012 to predict incident CVD risk after 2012 (Figure 1). Participants were excluded if they had MI or stroke prior to or in 2012, had missing data on FBG or TG at baseline or at all three measurements at 2008, 2010, and 2012, or with extreme outliers on the TyG index. Following these exclusions, we included 40,473 participants in the current analysis (Figure S1). The study was performed according to the guidelines of the Helsinki Declaration and was approved by the Ethics Committee of Kailuan General Hospital (approval number: 2006-05) and Beijing Tiantan Hospital (approval number: 2010-014-01). All the participants agreed to take part in the study and provided written informed consent.

\section{Data collection}

Demographic data, including age, sex, educational levels, and family income, lifestyle behaviors, including smoking, alcohol drinking and physical activity, as well as medical and medication history were collected via standardized questionnaires. Active physical activity was defined as physical activity $\geq 4$ times per week and $\geq 20$ minutes at a time. BMl was calculated as weight $(\mathrm{kg}) / \mathrm{height}(\mathrm{m})^{2}$. Blood pressure was measured in the seated position using a mercury sphygmomanometer, and the average of three measurements of the systolic blood pressure (SBP) and diastolic blood pressure (DBP) were recorded. All the blood samples were analyzed using an autoanalyzer (Hitachi 747, Hitachi, Tokyo, Japan) on the day of the blood draw. The biochemical indicators tested included FBG, serum lipids, serum creatinine, and high-sensitivity C-reactive protein (hs-CRP).

Hypertension was defined as SBP $\geq 140 \mathrm{~mm} \mathrm{Hg}$ or DBP $\geq 90 \mathrm{~mm} \mathrm{Hg}$, any use of the antihypertensive drug, or a self-reported history of hypertension. Diabetes was defined as FBG $\geq 7.0 \mathrm{mmol} / \mathrm{L}$, any use of glucose-lowering drugs, or a self-reported history of diabetes. Dyslipidemia was defined as any self-reported history or use of lipid-lowering drugs, or total cholesterol (TC) $\geq 5.17 \mathrm{mmol} / \mathrm{L}$.

\section{Assessment of the TyG index}

The TyG index was calculated as In (fasting TG [mg/dl] × FBG [mg/dl]/2), as descried previously.[26-28] The TyG index trajectories during 2006 to 2012 were the primary exposure of the current study. As secondary exposure, the annual increase of the TyG index (TyG index slope) was calculated using linear regression model in which the TyG index in 2006, 2008, 2010, and 2012 was the response variable and follow-up duration (years) was the independent variable.

\section{Assessment of incident CVD}

The outcome in our study were the first occurrence of CVD. The types of CVD included total stroke, ischemic stroke (IS), hemorrhagic stroke (HS), and myocardial infarction (MI). We used ICD-10th revision codes to identify CVD cases (I63 for IS, I60-161 for HS, and I21 for MI). The database of CVD diagnosed was obtained from the Municipal Social Insurance Institution and Hospital Discharge Register and was updated annually during the follow-up period. An expert panel collected and reviewed annual discharge records from 11 local hospitals to identify patients who were suspected of CVD. Incident stroke was diagnosed based on neurological signs, clinical symptoms, and neuroimaging tests, including computed tomography or magnetic resonance, according to the World Health Organization criteria.[29] MI was diagnosed according to the criteria of the World Health Organization on the based on the clinical symptoms, changes in the serum concentrations of cardiac enzymes and biomarkers, and electrocardiographic results.[16, 30]

\section{Statistical analysis}

The TyG index trajectories during 2006 to 2012 were identified using latent mixture modeling within the PROC TRAJ procedure in SAS. [31] We used a censored normal model appropriate for continuous outcomes. We initiated a model with 1 trajectory and then added 2, 3, 4 and up to 5 trajectory patterns. Model fit was assessed using the Bayesian information criterion (BIC), with the smallest negative number indicating the best fit model. We then compared the model with different functional forms. Cubic, quadratic, and linear terms 
were considered and evaluated based on their significance level, starting with the highest polynomial. In our final model, we had two patterns with linear order terms and three patterns with up to quadratic order terms.

Baseline characteristics were described as mean with standard deviation for continuous variables and frequency with percentage for categorical variables. Group differences were compared with Kruskal-Wallis test and chi-square test. The person-years were determined from the date when the message was collected at baseline to either the date of CVD diagnosis, death, or the end of the follow-up (December 31, 2019), whichever came first. The CVD and its subtypes probabilities were estimated by Kaplan-Meier method and the differences among groups were evaluated by log-rank test.

Cox proportional hazards model was used to investigate the association between exposures (the TyG index trajectories and annual increase rate of the TyG index from 2006 to 2012) and risk of developing CVD. According to Schoenfeld residuals and log-log inspection, the models met the proportional assumption criteria. Three models were built: model 1 was adjusted for age and sex; model 2 was further adjusted for education, income, smoking status, drinking status, physical activity, BMI, SBP, DBP, high density lipoprotein cholesterol (HDL-C), low density lipoprotein cholesterol (LDL-C) and hs-CRP; and model 3 was further adjusted for history of hypertension, diabetes, dyslipidemia, use of antihypertensive agents, antidiabetic agents, and lipid-lowering agents.

For more detailed of the association between annual increase rate of the TyG index from 2006 to 2012 and subsequent CVD risk, we also used restricted cubic spline models with 4 knots defined at the $5^{\text {th }}, 35^{\text {th }}, 65^{\text {th }}$, and $95^{\text {th }}$ percentiles of the TyG index.[28] Several sensitivity analyses were performed to validate the robustness of the results. First, we further adjusted the TyG index at baseline to control the regression-to-the mean influence. Second, we performed competing risk model considering non-CVD deaths as competing risk events. Third, we excluded participants who developed CVD cases within the first two years of follow up to minimize potential reverse causation. Likelihood ratio tests were conducted to examine statistical interactions between TyG index trajectories and age ( $<60$ vs. $\geq 60$ years), sex, hypertension (yes vs. no), diabetes (yes vs. no), and dyslipidemia (yes vs. no) in relation to CVD risk, by comparing -2 log likelihood c2 between nested models with and without the cross-product terms.

All analyses were conducted using SAS version 9.4 (SAS Institute Inc., Cary, NC, USA). A two-sided $P<0.05$ was considered statistically significant.

\section{Results}

\section{Baseline characteristics}

A total of 40,473 participants were selected for our analysis from 49,749 individuals with normal weight at baseline. A comparison of baseline characteristics between the included and excluded subjects due to missing the TyG index is shown in Table S1. Among the included participants, the mean age was $49.65 \pm 12.29$ years, and $30,427(75.18 \%)$ participants were men. Five discrete trajectories of the TyG index during 2006 and 2012 were identified based on the TyG index levels and changing pattern (Figure 2): low-stable ( $n=9,806$; mean TyG index ranged from 7.84 to 7.93 ), moderate-stable ( $n=22,066$; mean TyG index ranged from 8.43 to 8.52), elevated-decreasing $(n=1,469$; mean TyG index ranged from 9.83 to 8.75$)$, moderate-increasing ( $n=5,842$; mean TyG index ranged from 8.98 to 9.26$)$, and elevated-stable ( $n=1,290$; mean TyG index ranged from 9.91 to 10.07). Compared with participants in the low-stable pattern, participants in other patterns were older, more likely to be men, have lower education, income, more current smokers, current drink alcoholic beverages, take more active physical activity, have a higher prevalence of hypertension, diabetes, dyslipidemia, a higher proportion of participants taking antihypertensive, antidiabetic, and lipid-lowering agents, a higher level of BMI, SBP, DBP, TC, LDL-C and hs-CRP (Table 1).

\section{Association between the TyG index trajectories and CVD}

During a median follow-up of 6.74 years, we identified 1,577 cases of CVD, including 1,163 IS, 136 HS, and 306 MI. The elevated-stable TyG index pattern experienced the highest future risk of developing CVD among all five TyG index patterns $(P<0.0001$ for log-rank test; Figure 3), with an incidence rate of 13.00 (95\% confidence interval [Cl] 10.70-15.70) per 1000 person-years (Table 2). After adjustment for potential confounders, the adjusted hazard ratios (HRs) were $1.25(95 \% \mathrm{Cl}, 1.08-1.44)$ for the moderate-pattern, $1.45(95 \% \mathrm{Cl}, 1.11$ 1.89) for elevated-decreasing pattern, $1.70(95 \% \mathrm{Cl}, 1.43-2.04)$ for moderate-increasing pattern, and $2.24(95 \% \mathrm{Cl}, 1.73-2.90)$ for elevatedstable pattern, compared with low-stable pattern (Table 2). 
The results were not materially changed in the sensitivity analysis by further adjusted for the TyG index at baseline or the TyG index slope during the exposure period, with competing risk model, or excluding the outcome events that occurred within the first 2 years of the follow-up period (Table 2). Furthermore, we did not observe significant interaction between the TyG index trajectories and age, sex, hypertension, diabetes, and dyslipidemia in relation to CVD risk, the $P$ values for interaction were $>0.1$ for all (Table S2). Additionally, in the subtypes analyses of CVD, similar results were yielded for stroke, IS, and MI. However, most of the HRs in HS were not statistically significant, which could be limited by the relatively sample size of cases (Table 3).

\section{Association between annual increase of the TyG index and CVD}

Consistently, we observed that the annual increment of the TyG index was significantly associated with risk of developing CVD. The adjusted HR for CVD was 1.46 (95\% Cl, 1.23-1.73) for the highest quintile group, compared with the lowest quintile group (Table 4). Additionally, per 1 unit increase per year was associated with 1.99-fold higher risk of CVD (HR, 1.99; 95\% Cl, 1.41-2.83). In the restricted cubic spline models, we observed a J-shaped relationship between annual increment of the TyG index and CVD risk (Figure S2). Similar results were observed for stroke, IS, and MI (Table S3).

\section{Discussion}

In this prospective cohort study among normal-weight adults, we identified 5 distinct TyG index trajectories, in which participants shared a similar pattern of change in the TyG index during a 6-years exposure period. Participants with elevated-stable pattern had the highest risk of incident CVD whereas participants with low-stable pattern had the lowest risk of incident CVD. Notably, compared with the lowstable pattern, who started with a high TyG index and decreased substantially, and those who started with a moderate TyG index and increased substantially also had an increased risk of incident CVD. Furthermore, the risk of CVD tended to be higher for individuals with the moderate-increasing pattern than those with the elevated-decreasing pattern. The trends remained robust among the multiple sensitivity analyses and stratified analyses. These findings suggest that monitoring trajectories of TyG index may provide an important approach to identify a population with a higher risk of CVD and help to prevent primary CVD in a population with normal weight.

Many observational studies demonstrated that more than $30 \%$ of normal-weight people have metabolic abnormalities (MONW-like phenotype), including abdominal fat accumulation and IR. Subjects with the MONW phenotype are definitely regarded as a high-risk group among the normal weight population.[17-19] Results from the Whitehall II study showed that the incidence of CVD for MONW people was 12.20/1000 person-years, and the risk of incident CVD was more than two-fold increased as compared with metabolically health and normal weight people.[17] Similar results were observed in a Korean study, which showed the risk or incident CVD among MONW participants was 1.69-fold for men and 2.55-fold for women.[19] However, strategies to prevent CVD in normal weight people are lacking up to date. Our results provide evidence that the long-term TyG index trajectory patterns can be an important indicator for predicting CVD among normal-weight people, participants with distinct TyG index patterns presented different risk of CVD.

The present analyses showed that normal-weight adults with increasing and stable high TyG index level over time had a higher risk of CVD, compared with their counterparts with stable lower TyG index. The role of the TyG index in the development of CVD has been scarcely among normal weight adults. One study using data from the Korea National Health and Nutrition Examination Survey generated similar results that a higher level of TyG index at a single measurement predicted the risk of CVD in both men and women with normal weight (BMI $\geq 18.5$ and $<25.0 \mathrm{~kg} / \mathrm{m}^{2}$ ). [19] Of note, because of regression dilution, the study based on a single measurement of TyG index may underestimate the true association between the TyG index and CVD risk. To our knowledge, long-term patterns of the TyG index and their associations with the incidence of CVD are poorly characterized. Several studies, such as the Rural Chinese Cohort Study[32], the Chungju Metabolic Disease Cohort study[33], and the Vascular Metabolic CUN cohort study[34] demonstrated that changes in TyG index are determinant for forecasting diabetes among non-obese people. However, the classification of changes in TyG index was defined on the basis of 2 time points and were unable to account for potential fluctuations between the 2 measurements of the TyG index and thus oversimplified the heterogeneous and complex pattern of change in TyG index over time. By contrast, the present study used latent mixture modeling, which has several advantages over the approach used in the above study, with respect to the evaluation of indictor change. This approach could estimate the average, variability, and direction of variability simultaneously in one model and allow us to investigate the population heterogeneity in longitudinal changes in TyG index, which may provide additional information.[35]

In our analysis, we also found that individuals in the elevated-decreasing pattern had a higher risk of CVD than those in the moderatestable group, although they had similar TyG index levels at the end of the exposure period. This was in line with our previous publication 
that the risk of MI was increased with the times of high TyG index presented during the exposure period.[26] In addition, participants with the moderate-increasing pattern exhibited the cumulative average of TyG index similar to that exhibited by those with the elevateddecreasing pattern during the exposure period, whereas the risk of CVD was higher in moderate-increasing group than that in the elevated-decreasing group. The notion is further supported by the observation that participants with a higher annual increase of TyG index experienced a higher risk of CVD than those with a lower annual increase of TyG index. These findings indicate that a rapid increase in TyG index before the onset of disease contributed more to the pathological process of CVD, which emphasize the importance of maintaining a stable-low level of TyG index in the prevention of CVD.

The biological mechanisms underlying the association between TyG index trajectories and CVD have not been fully understood, several speculations summarized as follows. First, it has been demonstrated that the TyG index is closely related to traditional risk factors for CVD, such as hypertension.[36, 37] In the present study, participants with elevated TyG index patterns exactly tended to combine with more severe and complex clinical conditions in terms of BMI, blood pressure, lipids profiles, and hs-CRP. Second, study have shown that FBG mainly reflects IR from liver, whereas fasting TGs mainly reflects IR from adipose cell.[8, 38] Therefore, it can be concluded that TyG index may reflect IR from two aspects and thus be closely related to IR, which has been widely demonstrated to have significant relationship with endothelial dysfunction, oxidative stress, cardiovascular remodeling, coagulation imbalance and inflammation response. $[6,39,40]$ Third, the TyG index is related to arterial stiffness and coronary artery calcification through effects on platelet adhesion, activation, and aggregation, which has been recognized as cardiovascular risk predctor[41, 42].

Our study has several strengths. We conducted this study in a large prospective community cohort and put great emphasis on data quality. The entire study population was covered by biennial medical examinations and medical information enquiries, which enable us to collect precise data on TyG index repeatedly and track the outcome events in all participants. Statistical approach including the TyG index trajectories and annual increment were analyzed among normal-weight adults. The present study also has several potential limitations. First, we did not collect data on insulin concentrations, thus IR could not be evaluated by homeostasis model assessment of IR (HMOA-IR), which was not common practice on primary care. Second, we only collected information on stroke and MI, and we may have underestimated the prevalence and incidence rates of CVD, which has broader subtypes (e.g., heart failure, coronary artery disease). Third, the use of the latent class analysis has some weaknesses. For example, the method creates subgroups with very different sizes, making it difficult to compare subgroups in terms of statistical power. Moreover, while the participants in the same group tend to be homogenous, some individual variation around the group mean is allowed. However, because TyG index estimates are not static but change with time depending on the age and lifestyle of individuals, the latent class trajectory analysis used in our study is useful to explore the heterogeneous growth patterns because it is more flexible and models group specific average patterns of TyG index changes during follow-up. Finally, our study population included only normal-weight Chinese adults, and the trajectories identified in this population may not be generalizable to other populations. However, the biological effects of high TyG index on cardiovascular health in this cohort should be the same as those among men and women in general. The homogeneous nature of our cohort could help to reduce potential confounding due to racial and health care disparities and, therefore, enhance internal validity, which is a prerequisite for the generalizability.

\section{Conclusions}

We identified five distinct TyG index trajectories over 6 years and found that these patterns were significantly associated with subsequent risk of CVD in normal-weight adults. Our findings indicated that monitoring trajectories of TyG index may provide an important approach to identify a population with higher risk of $\mathrm{Ml}$ and help to prevent primary CVD in a population with normal weight.

\section{List Of Abbreviations}

$\mathrm{BIC}=$ Bayesian information criterion; $\mathrm{BMI}=$ body mass index; $\mathrm{Cl}=$ confidence interval; $\mathrm{CVD}=$ cardiovascular disease; $\mathrm{DBP}=$ diastolic blood pressure; $\mathrm{FBG}=$ fasting blood glucose; HDL-C=high-density lipoprotein cholesterol; HR=hazard ratio; HS=hemorrhagic stroke; hs$\mathrm{CRP}=$ high-sensitivity $\mathrm{C}$-reactive protein; $\mathrm{HMOA}-\mathrm{IR}=$ homeostasis model assessment of IR; IR=insulin resistance; IS=ischemic stroke; LDL$\mathrm{C}=$ =low-density lipoprotein cholesterol; $\mathrm{SBP}=$ systolic blood pressure; $\mathrm{TC}=$ total cholesterol; $\mathrm{TG}=$ triglyceride; $\mathrm{TyG}=$ triglyceride-glucose.

\section{Declarations}

\section{Ethics approval and consent to participate}


The study was performed according to the guidelines of the Helsinki Declaration and was approved by the Ethics Committee of Kailuan General Hospital (approval number: 2006-05) and Beijing Tiantan Hospital (approval number: 2010-014-01). All participants were agreed to take part in the study and provided informed written consent.

\section{Consent for publication}

Not applicable

\section{Availability of data and materials}

The datasets used and/or analyzed during the current study are available from the corresponding author on reasonable request

\section{Competing interests}

These authors declare that they have no conflicts of interests.

\section{Funding}

This work was supported by grants from National Key R\&D Program of China (2018YFC1312903), National Natural Science Foundation of China (81870905, 81773512), Beijing Municipal Science \& Technology Commission (D171100003017002, Z181100001818001), Beijing Municipal Administration of Hospitals Incubating Program (PX2020021), Beijing Excellent Talents Training Program (2018000021469G234), and Young Elite Scientists Sponsorship Program by CAST (2018QNRC001).

\section{Author Contributions}

S.W., Y.L. and Y.W. contributed to the conception and design of the study; A.W. and X.T. contributed to manuscript drafting; A.W., X.T., Y.Z. and S.C. contributed to the statistics analysis; S.C., X.M. and P.C. contributed to the acquisition of data; S.W., Y.L. and Y.W. contributed to critical revisions of the manuscript. All authors read and approved the final manuscript.

\section{Acknowledgments}

We thank all study participants, their relatives, the members of the survey teams at the 11 regional hospitals of the Kailuan Medical Group; and the project development and management teams at the Beijing Tiantan Hospital and the Kailuan Group.

\section{References}

1. Xun P, Wu Y, He Q, He K. Fasting insulin concentrations and incidence of hypertension, stroke, and coronary heart disease: a metaanalysis of prospective cohort studies. Am J Clin Nutr. 2013;98(6):1543-54.

2. Hildreth KL, Van Pelt RE, Schwartz RS. Obesity, insulin resistance, and Alzheimer's disease. Obesity (Silver Spring). 2012;20(8):1549-57.

3. Baghbani-Oskouei A, Tohidi M, Hasheminia M, Azizi F, Hadaegh F. Impact of 3-year changes in fasting insulin and insulin resistance indices on incident hypertension: Tehran lipid and glucose study. Nutrition metabolism. 2019;16:76.

4. Kozakova M, Natali A, Dekker J, Beck-Nielsen H, Laakso M, Nilsson P, Balkau B, Ferrannini E. Insulin sensitivity and carotid intimamedia thickness: relationship between insulin sensitivity and cardiovascular risk study. Arterioscler Thromb Vasc Biol. 2013;33(6):1409-17.

5. Pan K, Nelson RA, Wactawski-Wende J, Lee DJ, Manson JE, Aragaki AK, Mortimer JE, Phillips LS, Rohan T, Ho GYF, et al. Insulin Resistance and Cancer-Specific and All-Cause Mortality in Postmenopausal Women: The Women's Health Initiative. J Natl Cancer Inst. 2020;112(2):170-8.

6. Ormazabal V, Nair S, Elfeky O, Aguayo C, Salomon C, Zuñiga FA. Association between insulin resistance and the development of cardiovascular disease. Cardiovasc Diabetol. 2018;17(1):122.

7. Zhou M, Wang H, Zeng X, Yin P, Zhu J, Chen W, Li X, Wang L, Wang L, Liu Y, et al. Mortality, morbidity, and risk factors in China and its provinces, 1990-2017: a systematic analysis for the Global Burden of Disease Study 2017. Lancet. 2019;394(10204):1145-58.

8. Zhao Q, Zhang TY, Cheng YJ, Ma Y, Xu YK, Yang JQ, Zhou YJ. Impacts of triglyceride-glucose index on prognosis of patients with type 2 diabetes mellitus and non-ST-segment elevation acute coronary syndrome: results from an observational cohort study in China. Cardiovasc Diabetol. 2020;19(1):108. 
9. Guerrero-Romero F, Simental-Mendía LE, González-Ortiz M, Martínez-Abundis E, Ramos-Zavala MG, Hernández-González SO, Jacques-Camarena $\mathrm{O}$, Rodríguez-Morán $\mathrm{M}$. The product of triglycerides and glucose, a simple measure of insulin sensitivity. Comparison with the euglycemic-hyperinsulinemic clamp. J Clin Endocrinol Metab. 2010;95(7):3347-51.

10. Du T, Yuan G, Zhang M, Zhou X, Sun X, Yu X. Clinical usefulness of lipid ratios, visceral adiposity indicators, and the triglycerides and glucose index as risk markers of insulin resistance. Cardiovasc Diabetol. 2014;13:146.

11. Barzegar N, Tohidi M, Hasheminia M, Azizi F, Hadaegh F. The impact of triglyceride-glucose index on incident cardiovascular events during 16 years of follow-up: Tehran Lipid and Glucose Study. Cardiovasc Diabetol. 2020;19(1):155.

12. Sánchez-Íñigo L, Navarro-González D, Fernández-Montero A, Pastrana-Delgado J, Martínez J. The TyG index may predict the development of cardiovascular events. European journal of clinical investigation. 2016;46(2):189-97.

13. Luo E, Wang D, Yan G, Qiao Y, Liu B, Hou J, Tang C. High triglyceride-glucose index is associated with poor prognosis in patients with acute ST-elevation myocardial infarction after percutaneous coronary intervention. Cardiovasc Diabetol. 2019;18(1):150.

14. Ding X, Wang X, Wu J, Zhang M, Cui M. Triglyceride-glucose index and the incidence of atherosclerotic cardiovascular diseases: a meta-analysis of cohort studies. Cardiovasc Diabetol. 2021;20(1):76.

15. Li S, Guo B, Chen H, Shi Z, Li Y, Tian Q, Shi S. The role of the triglyceride (triacylglycerol) glucose index in the development of cardiovascular events: a retrospective cohort analysis. Scientific reports. 2019;9(1):7320.

16. Jin C, Chen S, Vaidya A, Wu Y, Wu Z, Hu FB, Kris-Etherton P, Wu S, Gao X. Longitudinal Change in Fasting Blood Glucose and Myocardial Infarction Risk in a Population Without Diabetes. Diabetes Care. 2017;40(11):1565-72.

17. Hinnouho GM, Czernichow S, Dugravot A, Nabi H, Brunner EJ, Kivimaki M, Singh-Manoux A. Metabolically healthy obesity and the risk of cardiovascular disease and type 2 diabetes: the Whitehall II cohort study. European heart journal. 2015;36(9):551-9.

18. Aung K, Lorenzo C, Hinojosa MA, Haffner SM. Risk of developing diabetes and cardiovascular disease in metabolically unhealthy normal-weight and metabolically healthy obese individuals. J Clin Endocrinol Metab. 2014;99(2):462-8.

19. Lee SH, Han K, Yang HK, Kim HS, Cho JH, Kwon HS, Park YM, Cha BY, Yoon KH. A novel criterion for identifying metabolically obese but normal weight individuals using the product of triglycerides and glucose. Nutr Diabetes. 2015;5(4):e149.

20. Nazare JA, Smith JD, Borel AL, Haffner SM, Balkau B, Ross R, Massien C, Alméras N, Després JP. Ethnic influences on the relations between abdominal subcutaneous and visceral adiposity, liver fat, and cardiometabolic risk profile: the International Study of Prediction of Intra-Abdominal Adiposity and Its Relationship With Cardiometabolic Risk/Intra-Abdominal Adiposity. Am J Clin Nutr. 2012;96(4):714-26.

21. Gao H, Salim A, Lee J, Tai ES, van Dam RM. Can body fat distribution, adiponectin levels and inflammation explain differences in insulin resistance between ethnic Chinese, Malays and Asian Indians? Int J Obes (Lond). 2012;36(8):1086-93.

22. Wang A, Sun Y, Liu X, Su Z, Li J, Luo Y, Chen S, Wang J, Li X, Zhao Z, et al. Changes in proteinuria and the risk of myocardial infarction in people with diabetes or pre-diabetes: a prospective cohort study. Cardiovascular diabetology. 2017;16(1):104.

23. Wang L, Lee Y, Wu Y, Zhang X, Jin C, Huang Z, Wang Y, Wang Z, Kris-Etherton P, Wu S, et al. A prospective study of waist circumference trajectories and incident cardiovascular disease in China: the Kailuan Cohort Study. Am J Clin Nutr. 2021;113(2):338-47.

24. Wu S, An S, Li W, Lichtenstein AH, Gao J, Kris-Etherton PM, Wu Y, Jin C, Huang S, Hu FB, et al. Association of Trajectory of Cardiovascular Health Score and Incident Cardiovascular Disease. JAMA network open. 2019;2(5):e194758.

25. Yang S, Liu M, Wang S, Jia W, Han K, He Y. Waist-Calf Circumference Ratio Is an Independent Risk Factor of HRQoL in Centenarians. Diabetes metabolic syndrome obesity: targets therapy. 2020;13:277-87.

26. Tian X, Zuo Y, Chen S, Liu Q, Tao B, Wu S, Wang A. Triglyceride-glucose index is associated with the risk of myocardial infarction: an 11-year prospective study in the Kailuan cohort. Cardiovasc Diabetol. 2021;20(1):19.

27. Wang A, Wang G, Liu Q, Zuo Y, Chen S, Tao B, Tian X, Wang P, Meng X, Wu S, et al. Triglyceride-glucose index and the risk of stroke and its subtypes in the general population: an 11-year follow-up. Cardiovasc Diabetol. 2021;20(1):46.

28. Wang A, Tian X, Zuo Y, Chen S, Meng X, Wu S, Wang Y. Change in triglyceride-glucose index predicts the risk of cardiovascular disease in the general population: a prospective cohort study. Cardiovasc Diabetol. 2021;20(1):113.

29. Stroke-1989. Recommendations on stroke prevention, diagnosis, and therapy. Report of the WHO Task Force on Stroke and other Cerebrovascular Disorders. Stroke. 1989;20(10):1407-31.

30. Tunstall-Pedoe H, Kuulasmaa K, Amouyel P, Arveiler D, Rajakangas A, Pajak A. Myocardial infarction and coronary deaths in the World Health Organization MONICA Project. Registration procedures, event rates, and case-fatality rates in 38 populations from 21

Page $8 / 15$ 
countries in four continents. Circulation. 1994;90(1):583-612.

31. Jones BLND. Advances in group-based trajectory modeling.

32. and an SAS procedure for estimating them. Sociol Methods Res. 2007, 35:542-571.

33. Zhang M, Wang B, Liu Y, Sun X, Luo X, Wang C, Li L, Zhang L, Ren Y, Zhao Y, et al. Cumulative increased risk of incident type 2 diabetes mellitus with increasing triglyceride glucose index in normal-weight people: The Rural Chinese Cohort Study. Cardiovasc Diabetol. 2017;16(1):30.

34. Lee SH, Yang HK, Ha HS, Lee JH, Kwon HS, Park YM, Yim HW, Kang MI, Lee WC, Son HY, et al. Changes in Metabolic Health Status Over Time and Risk of Developing Type 2 Diabetes: A Prospective Cohort Study. Medicine. 2015;94(40):e1705.

35. Navarro-González D, Sánchez-Iñigo L, Fernández-Montero A, Pastrana-Delgado J, Martinez JA. TyG Index Change Is More Determinant for Forecasting Type 2 Diabetes Onset Than Weight Gain. Medicine. 2016;95(19):e3646.

36. Ma H, Wang X, Guo X, Li X, Qi L, Li Y. Distinct Uric Acid Trajectories Are Associated With Different Risks of Incident Hypertension in Middle-Aged Adults. Mayo Clinic proceedings. 2019, 94(4):611-619.

37. Francula-Zaninovic S, Nola IA. Management of Measurable Variable Cardiovascular Disease' Risk Factors. Curr Cardiol Rev. 2018;14(3):153-63.

38. Cortesi P, Fornari C, Madotto F, Conti S, Naghavi M, Bikbov B, Briant P, Caso V, Crotti G, Johnson C, et al. Trends in cardiovascular diseases burden and vascular risk factors in Italy: The Global Burden of Disease study 1990-2017. European journal of preventive cardiology. 2020:2047487320949414.

39. Low S, Khoo K, Irwan B, Sum C, Subramaniam T, Lim S, Wong T. The role of triglyceride glucose index in development of Type 2 diabetes mellitus. Diabetes Res Clin Pract. 2018;143:43-9.

40. Janus A, Szahidewicz-Krupska E, Mazur G, Doroszko A. Insulin Resistance and Endothelial Dysfunction Constitute a Common Therapeutic Target in Cardiometabolic Disorders. Mediators of inflammation. 2016, 2016:3634948.

41. Markus MRP, Rospleszcz S, Ittermann T, Baumeister SE, Schipf S, Siewert-Markus U, Lorbeer R, Storz C, Ptushkina V, Peters A, et al. Glucose and insulin levels are associated with arterial stiffness and concentric remodeling of the heart. Cardiovasc Diabetol. 2019;18(1):145.

42. Zhao S, Yu S, Chi C, Fan X, Tang J, Ji H, Teliewubai J, Zhang Y, Xu Y. Association between macro- and microvascular damage and the triglyceride glucose index in community-dwelling elderly individuals: the Northern Shanghai Study. Cardiovasc Diabetol. 2019;18(1):95.

43. Won K, Park G, Lee S, Cho I, Kim H, Lee B, Chang H. Relationship of insulin resistance estimated by triglyceride glucose index to arterial stiffness. Lipids Health Dis. 2018;17(1):268.

\section{Tables}

Table 1. Baseline characteristics of participants according to trajectories of the TyG index 


\begin{tabular}{|c|c|c|c|c|c|c|c|}
\hline \multirow[b]{2}{*}{ Characteristics } & \multirow[b]{2}{*}{ Overall } & \multicolumn{6}{|c|}{ TyG index trajectories } \\
\hline & & Low-stable & $\begin{array}{l}\text { Moderate- } \\
\text { stable }\end{array}$ & $\begin{array}{l}\text { Elevated- } \\
\text { decreasing }\end{array}$ & $\begin{array}{l}\text { Moderate- } \\
\text { increasing }\end{array}$ & $\begin{array}{l}\text { Elevated- } \\
\text { stable }\end{array}$ & $P$ value \\
\hline $\begin{array}{l}\text { No. of } \\
\text { participants }\end{array}$ & 40473 & 9806 & 22066 & 1469 & 5842 & 1290 & \\
\hline Age, years & $49.65 \pm 12.29$ & $48.31 \pm 12.94$ & $50.06 \pm 12.40$ & $51.55 \pm 11.03$ & $49.89 \pm 11.3$ & $49.56 \pm 9.86$ & $<0.0001$ \\
\hline Men, n (\%) & $\begin{array}{l}30427 \\
(75.18)\end{array}$ & $6694(68.26)$ & $\begin{array}{l}16869 \\
(76.45)\end{array}$ & $1236(84.14)$ & 4556 (77.99) & $1072(83.10)$ & $<0.0001$ \\
\hline $\begin{array}{l}\text { High school or } \\
\text { above, } \mathrm{n}(\%)\end{array}$ & $3251(8.27)$ & $1138(11.95)$ & $1590(7.425)$ & $46(3.18)$ & $408(7.20)$ & $69(5.58)$ & $<0.0001$ \\
\hline $\begin{array}{l}\text { Income }>800 \\
\text { yuan/month, } n \\
(\%)\end{array}$ & $5626(14.33)$ & $1466(15.39)$ & $2974(13.90)$ & $205(14.21)$ & $842(14.87)$ & 139 (11.22) & 0.0001 \\
\hline $\mathrm{BMI}, \mathrm{kg} / \mathrm{m}^{2}$ & $22.56 \pm 1.64$ & $21.97 \pm 1.70$ & $22.61 \pm 1.60$ & $22.99 \pm 1.52$ & $23.10 \pm 1.46$ & $23.30 \pm 1.36$ & $<0.0001$ \\
\hline SBP, mm Hg & $125.24 \pm 19.26$ & $120.62 \pm 18.34$ & $125.58 \pm 19.08$ & $133.16 \pm 20.49$ & $128.27 \pm 19.27$ & $131.77 \pm 19.44$ & $<0.0001$ \\
\hline $\mathrm{DBP}, \mathrm{mm} \mathrm{Hg}$ & $80.40 \pm 10.83$ & $77.74 \pm 10.32$ & $80.60 \pm 10.75$ & $84.25 \pm 10.74$ & $82.23 \pm 10.81$ & $84.37 \pm 11.33$ & $<0.0001$ \\
\hline $\begin{array}{l}\text { Current smoker, } \\
\mathrm{n}(\%)\end{array}$ & $\begin{array}{l}13909 \\
(35.19)\end{array}$ & $3032(31.73)$ & 7431 (34.47) & $559(38.50)$ & $2306(40.43)$ & $581(46.18)$ & $<0.0001$ \\
\hline $\begin{array}{l}\text { Current alcohol } \\
\text { use, } n(\%)\end{array}$ & $\begin{array}{l}15152 \\
(38.32)\end{array}$ & $3457(36.15)$ & $8006(37.14)$ & $613(42.19)$ & $2468(43.26)$ & $608(48.33)$ & $<0.0001$ \\
\hline $\begin{array}{l}\text { Active physical } \\
\text { activity, } \mathrm{n}(\%)\end{array}$ & $\begin{array}{l}35525 \\
(90.59)\end{array}$ & $8711(91.61)$ & $\begin{array}{l}19339 \\
(90.49)\end{array}$ & $1315(91.00)$ & $5057(89.46)$ & $1103(89.31)$ & 0.0001 \\
\hline $\begin{array}{l}\text { Hypertension, } \mathrm{n} \\
(\%)\end{array}$ & $\begin{array}{l}12805 \\
(31.64)\end{array}$ & $2112(21.54)$ & $7130(32.31)$ & $710(48.33)$ & $2241(38.36)$ & $612(47.44)$ & $<0.0001$ \\
\hline $\begin{array}{l}\text { Diabetes } \\
\text { Mellitus, } \mathrm{n}(\%)\end{array}$ & $2360(5.83)$ & $80(0.82)$ & $805(3.65)$ & $363(24.71)$ & $708(12.12)$ & $404(31.32)$ & $<0.0001$ \\
\hline $\begin{array}{l}\text { Dyslipidemia, n } \\
\text { (\%) }\end{array}$ & $\begin{array}{l}10913 \\
(26.96)\end{array}$ & $1216(12.40)$ & $4426(20.06)$ & $1318(89.72)$ & 2827 (48.39) & $1126(87.29)$ & $<0.0001$ \\
\hline $\begin{array}{l}\text { Antihypertensive } \\
\text { agents, } n(\%)\end{array}$ & $2254(5.57)$ & $306(3.12)$ & $1217(5.52)$ & $124(8.44)$ & $469(8.03)$ & $138(10.70)$ & $<0.0001$ \\
\hline $\begin{array}{l}\text { Antidiabetic } \\
\text { agents, n (\%) }\end{array}$ & $570(1.41)$ & $16(0.16)$ & $191(0.86)$ & $68(4.63)$ & $205(3.51)$ & $90(6.98)$ & $<0.0001$ \\
\hline $\begin{array}{l}\text { Lipid-lowering } \\
\text { agents, n (\%) }\end{array}$ & $196(0.48)$ & $14(0.14)$ & $100(0.45)$ & $10(0.68)$ & $56(0.96)$ & $16(1.24)$ & $<0.0001$ \\
\hline $\mathrm{FBG}, \mathrm{mmol} / \mathrm{L}$ & $5.27 \pm 1.46$ & $4.81 \pm 0.66$ & $5.16 \pm 0.99$ & $6.64 \pm 3.17$ & $5.72 \pm 1.84$ & $7.21 \pm 3.33$ & $<0.0001$ \\
\hline $\mathrm{TC}, \mathrm{mmol} / \mathrm{L}$ & $4.87 \pm 1.12$ & $4.59 \pm 0.89$ & $4.93 \pm 1.02$ & $4.04 \pm 2.13$ & $5.22 \pm 1.13$ & $5.50 \pm 1.36$ & $<0.0001$ \\
\hline $\begin{array}{l}\text { Triglyceride, } \\
\mathrm{mmol} / \mathrm{L}\end{array}$ & $1.42 \pm 1.20$ & $0.71 \pm 0.26$ & $1.22 \pm 0.52$ & $4.20 \pm 1.92$ & $2.00 \pm 0.91$ & $4.48 \pm 2.77$ & $<0.0001$ \\
\hline $\mathrm{HDL}-\mathrm{C}, \mathrm{mmol} / \mathrm{L}$ & $1.58 \pm 0.40$ & $1.59 \pm 0.40$ & $1.58 \pm 0.39$ & $1.63 \pm 0.43$ & $1.54 \pm 0.40$ & $1.55 \pm 0.47$ & $<0.0001$ \\
\hline LDL-C, mmol/L & $2.30 \pm 0.90$ & $2.12 \pm 0.90$ & $2.35 \pm 0.87$ & $2.37 \pm 0.86$ & $2.39 \pm 0.90$ & $2.26 \pm 1.04$ & $<0.0001$ \\
\hline Hs-CRP,mg/dL & $2.02 \pm 6.06$ & $1.96 \pm 5.85$ & $1.98 \pm 6.32$ & $2.11 \pm 5.49$ & $2.07 \pm 4.32$ & $2.84 \pm 9.45$ & $<0.0001$ \\
\hline
\end{tabular}

Abbreviations: BMI, body mass index; DBP, diastolic blood pressure; FBG, fasting blood glucose; LDL-C, low-density lipoprotein cholesterol; HDL-C, high-density lipoprotein cholesterol; hs-CRP, high-sensitivity C-reactive protein; SBP, systolic blood pressure; TC, total cholesterol; TyG, triglyceride glucose. 
Table 2. Association between the TyG index trajectories and risk of cardiovascular disease

\begin{tabular}{|c|c|c|c|c|c|}
\hline & \multicolumn{5}{|c|}{ TyG index trajectories from 2006-2010 } \\
\hline & Low-stable & Moderate-stable & Elevated-decreasing & Moderate-increasing & Elevated-stable \\
\hline Case, n (\%) & $250(2.55)$ & $820(3.72)$ & $88(5.99)$ & $313(5.36)$ & $106(8.22)$ \\
\hline Incidence rate* & $3.89(3.44-4.41)$ & $5.74(5.36-6.14)$ & $9.45(7.67-11.60)$ & 8.35(7.47-9.32) & $13.00(10.70-15.70)$ \\
\hline Model 1 & Reference & $1.37(1.19-1.58)$ & $2.14(1.68-2.73)$ & $2.12(1.80-2.51)$ & $3.43(2.73-4.31)$ \\
\hline Model 2 & Reference & $1.29(1.11-1.48)$ & $1.77(1.39-2.27)$ & $1.91(1.61-2.27)$ & $2.86(2.27-3.61)$ \\
\hline Model 3 & Reference & $1.25(1.08-1.44)$ & $1.45(1.11-1.89)$ & $1.70(1.43-2.04)$ & $2.24(1.73-2.90)$ \\
\hline \multicolumn{6}{|l|}{ Sensitivity analysis } \\
\hline Sensitivity analysis ${ }^{1}$ & Reference & $1.19(1.02-1.40)$ & $1.27(0.90-1.79)$ & $1.58(1.26-1.96)$ & $1.95(1.38-2.75)$ \\
\hline Sensitivity analysis ${ }^{2}$ & Reference & $1.24(1.08-1.44)$ & $1.41(1.08-1.85)$ & $1.69(1.41-2.02)$ & $2.20(1.70-2.85)$ \\
\hline Sensitivity analysis ${ }^{3}$ & Reference & $1.21(1.02-1.42)$ & $1.48(1.09-2.00)$ & $1.64(1.34-2.01)$ & $2.24(1.67-3.01)$ \\
\hline
\end{tabular}

Abbreviations: CVD, cardiovascular disease; TyG index, triglyceride-glucose index.

* Incidence rate per 1000 person-years.

Model 1 was adjusted for age and sex.

Model 2 was further adjusted for education, income, smoking status, drinking status, physical activity, body mass index, systolic blood pressure, diastolic blood pressure, high-density lipoprotein cholesterol, low-density lipoprotein cholesterol and high sensitivity C-reactive protein.

Model 3 was further adjusted for history of hypertension, diabetes, dyslipidemia, use of antihypertensive agents, antidiabetic agents, and lipid-lowering agents.

Sensitivity analysis ${ }^{1}$ was adjusted for variables in Model 3 and further adjusted for the TyG index at baseline.

Sensitivity analysis ${ }^{2}$ was used competing risk model considering death as a competing risk and adjusted for variables in Model 3.

Sensitivity analysis ${ }^{3}$ excluded outcome events within the first two year of follow-up and adjusted for variables in Model 3.

Table 3. Association with trajectories of TyG index with risk of cardiovascular disease subtypes 


\begin{tabular}{|c|c|c|c|c|c|}
\hline \multirow[b]{2}{*}{ Outcomes } & \multicolumn{5}{|c|}{ TyG index trajectories from 2006-2010 } \\
\hline & Low-stable & Moderate-stable & Elevated-decreasing & Moderate-increasing & Elevated-stable \\
\hline \multicolumn{6}{|l|}{ Total stroke } \\
\hline Case, n (\%) & $218(2.22)$ & $663(3.00)$ & $76(5.17)$ & $245(4.19)$ & $90(6.98)$ \\
\hline Incidence rate* & $3.39(2.97-3.87)$ & $4.62(4.28-4.99)$ & $8.12(6.49-10.2)$ & $6.5(5.73-7.36)$ & 11(8.91-13.5) \\
\hline Adjusted HR (95\% Cl) & Reference & $1.15(0.98-1.34)$ & $1.45(1.09-1.94)$ & $1.53(1.26-1.86)$ & $2.19(1.66-2.91)$ \\
\hline \multicolumn{6}{|l|}{ Ischemic stroke } \\
\hline Case, n (\%) & $182(1.86)$ & $602(2.73)$ & $73(4.97)$ & $224(3.83)$ & $82(6.36)$ \\
\hline Incidence rate* & $2.83(2.44-3.27)$ & $4.19(3.87-4.54)$ & 7.72(6.13-9.71) & $5.93(5.2-6.76)$ & $9.98(8.03-12.4)$ \\
\hline Adjusted HR $(95 \% \mathrm{Cl})$ & Reference & $1.24(1.05-1.47)$ & $1.65(1.22-2.23)$ & $1.67(1.35-2.06)$ & 2.39(1.78-3.22) \\
\hline \multicolumn{6}{|l|}{ Hemorrhagic stroke } \\
\hline Case, n (\%) & $33(0.34)$ & $65(0.29)$ & $4(0.27)$ & $25(0.43)$ & $9(0.70)$ \\
\hline Incidence rate* & $0.51(0.36-0.72)$ & $0.45(0.35-0.57)$ & $0.42(0.16-1.12)$ & $0.65(0.44-0.97)$ & $1.07(0.55-2.05)$ \\
\hline Adjusted HR $(95 \% \mathrm{Cl})$ & Reference & $0.79(0.51-1.21)$ & $0.61(0.20-1.87)$ & $1.22(0.69-2.15)$ & $1.81(0.77-4.29)$ \\
\hline \multicolumn{6}{|l|}{ Myocardial infarction } \\
\hline Case, n (\%) & $36(0.37)$ & $165(0.75)$ & $14(0.95)$ & $74(1.27)$ & $17(1.32)$ \\
\hline Incidence rate* & $0.56(0.4-0.77)$ & $1.14(0.98-1.33)$ & $1.47(0.87-2.49)$ & $1.94(1.54-2.44)$ & $2.02(1.26-3.25)$ \\
\hline Adjusted HR (95\% Cl) & Reference & $1.73(1.20-2.50)$ & $1.49(0.77-2.90)$ & $2.40(1.27-4.54)$ & $2.71(1.77-4.14)$ \\
\hline
\end{tabular}

Abbreviations: TyG index, triglyceride-glucose index.

* Incidence rate per 1000 person-years.

Adjusted for age, sex, education, income, smoking status, drinking status, physical activity, history of hypertension, diabetes, dyslipidemia, use of antihypertensive agents, antidiabetic agents, lipid-lowering agents, body mass index, systolic blood pressure, diastolic blood pressure, high-density lipoprotein cholesterol, low-density lipoprotein cholesterol and high sensitivity C-reactive protein.

Table 4. Association between annual increase of the TyG index and risk of cardiovascular disease

\begin{tabular}{|c|c|c|c|c|c|}
\hline & \multicolumn{5}{|c|}{ Annual increase of TyG index from 2006 to 2010} \\
\hline & Q1 & Q2 & Q3 & Q4 & Q5 \\
\hline Range, per 1 unit/year & $<-0.07$ & $-0.07 \sim-0.01$ & $-0.01 \sim 0.04$ & $0.04 \sim 0.10$ & $\geq 0.10$ \\
\hline Case, n (\%) & $265(3.27)$ & $285(3.52)$ & $311(3.84)$ & $343(4.24)$ & $373(4.61)$ \\
\hline Incidence rate* & $5.01(4.45-5.66)$ & $5.44(4.84-6.11)$ & $5.88(5.27-6.58)$ & $6.58(5.92-7.32)$ & 7.18(6.49-7.95) \\
\hline Model 1 & Reference & $1.19(1.00-1.41)$ & $1.20(1.02-1.42)$ & $1.28(1.10-1.49)$ & $1.48(1.25-1.75)$ \\
\hline Model 2 & Reference & $1.22(1.03-1.45)$ & $1.23(1.04-1.45)$ & $1.30(1.11-1.51)$ & $1.49(1.26-1.77)$ \\
\hline Model 3 & Reference & $1.21(1.02-1.44)$ & $1.22(1.04-1.45)$ & $1.30(1.12-1.52)$ & $1.46(1.23-1.73)$ \\
\hline
\end{tabular}

Abbreviations: CVD, cardiovascular disease; TyG index, triglyceride-glucose index.

* Incidence rate per 1000 person-years.

Model 1 was adjusted for age, sex and baseline TyG index. 
Model 2 was further adjusted for education, income, smoking status, drinking status, physical activity, body mass index, systolic blood pressure, diastolic blood pressure, high-density lipoprotein cholesterol, low-density lipoprotein cholesterol and high sensitivity C-reactive protein.

Model 3 was further adjusted for history of hypertension, diabetes, dyslipidemia, use of antihypertensive agents, antidiabetic agents, and lipid-lowering agents.

\section{Figures}

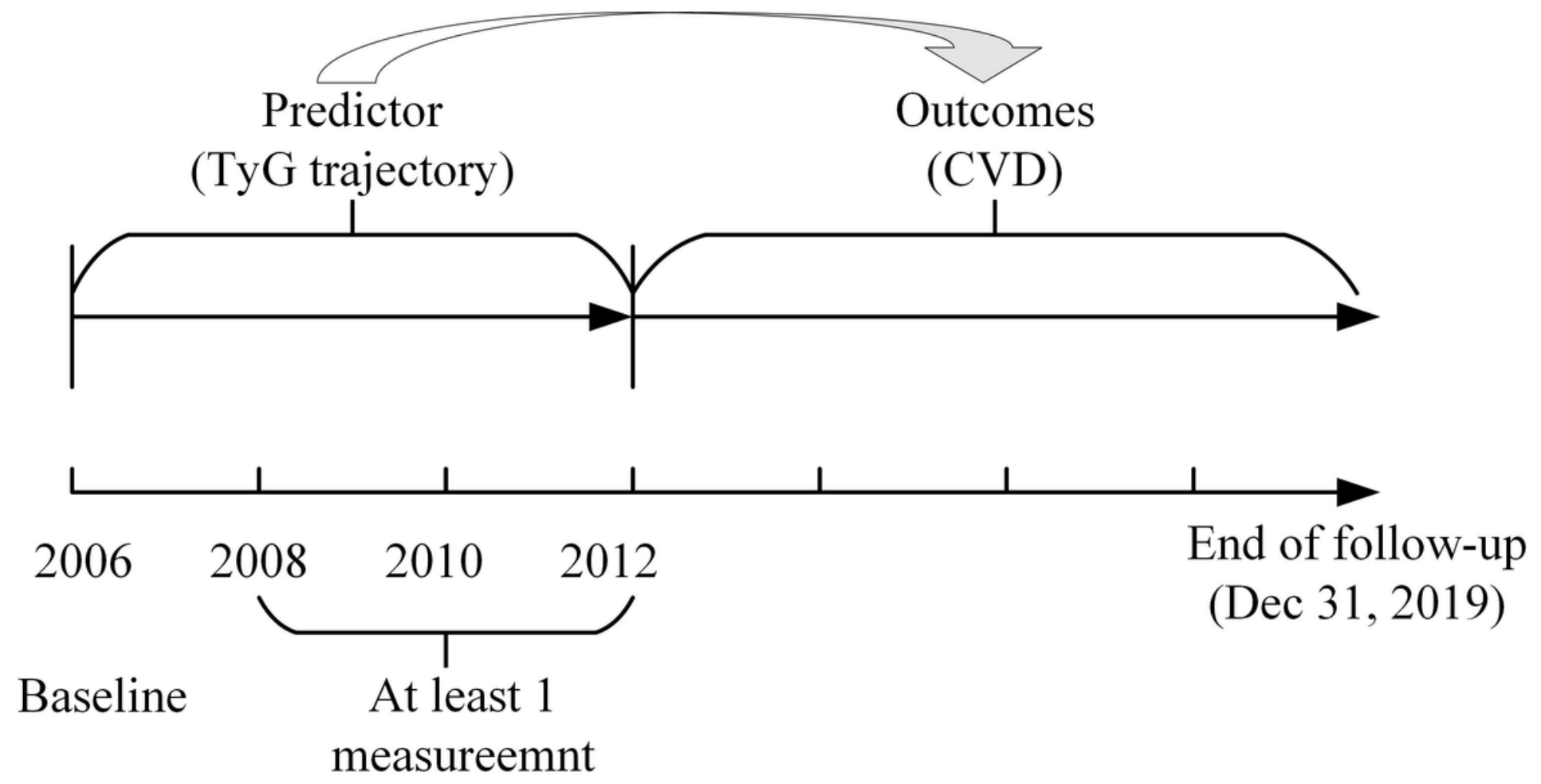

\section{Figure 1}

Time line of exposure and follow-up assessment. Abbreviations: CVD, cardiovascular disease; TyG index, triglyceride-glucose index 


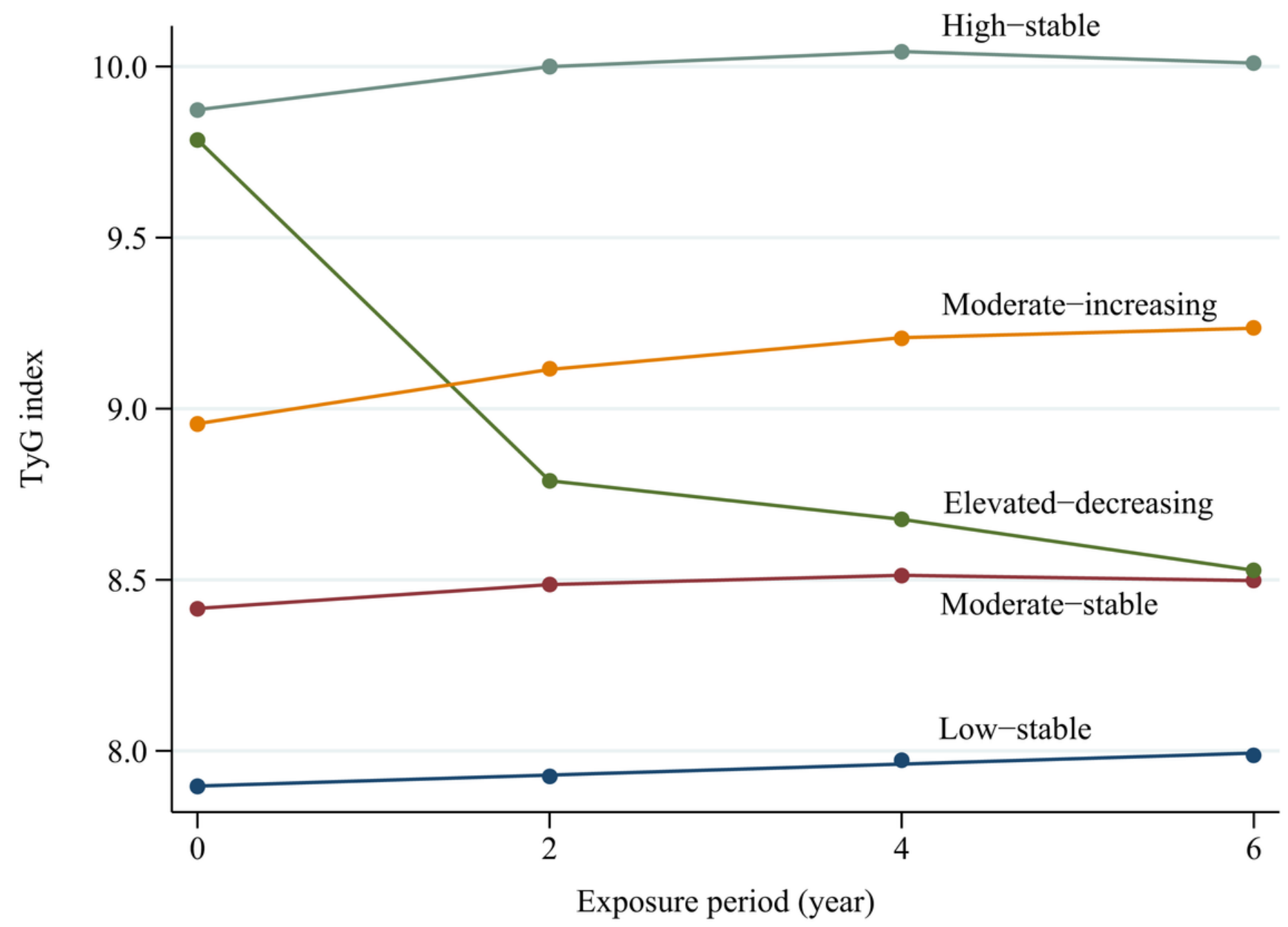

Figure 2

Trajectory of the TyG index during 2006-2012. Abbreviations: TyG index, triglyceride-glucose index 


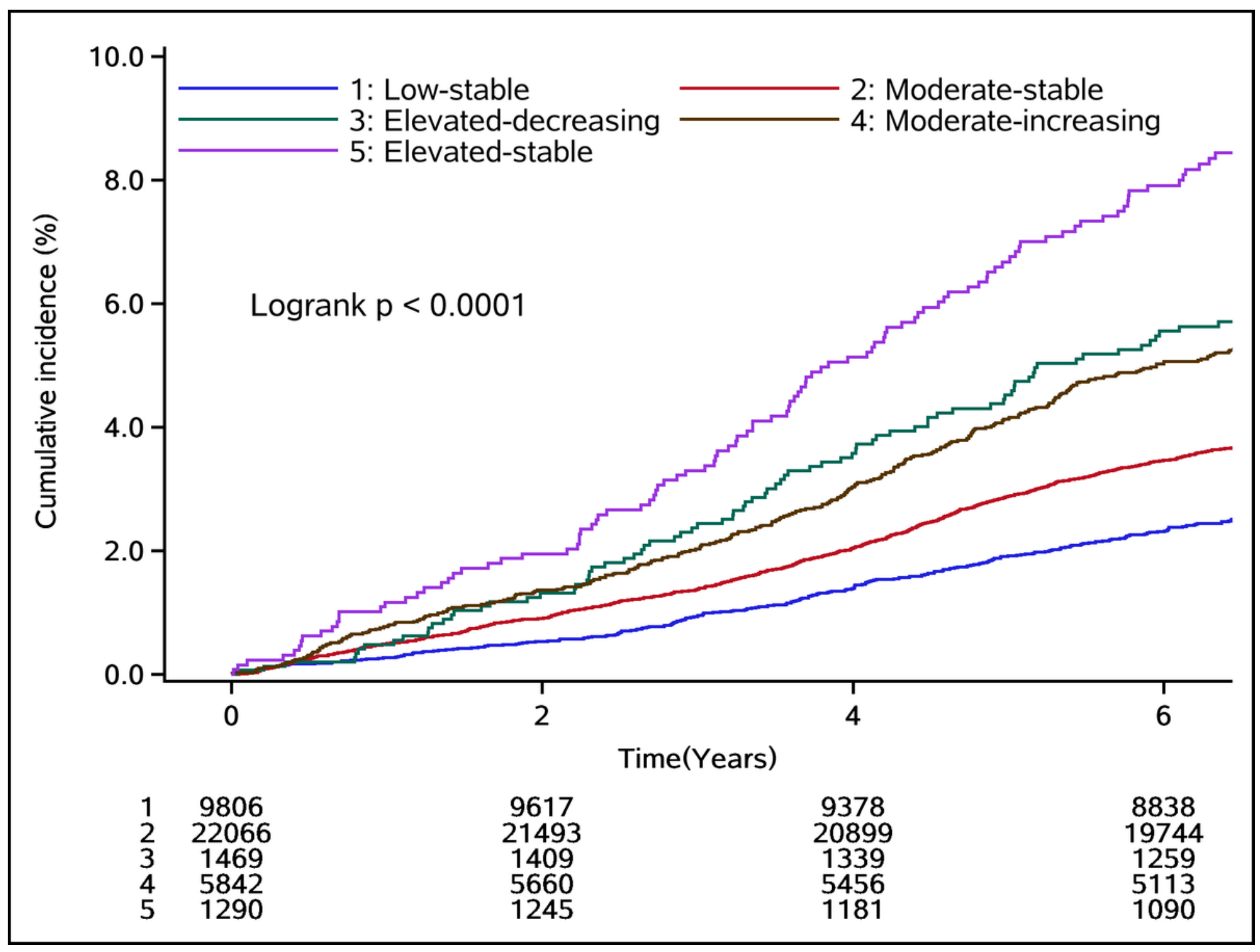

Figure 3

Kaplan-Meier estimation of cardiovascular diseases by TyG index trajectories during 2006-2012.

\section{Supplementary Files}

This is a list of supplementary files associated with this preprint. Click to download.

- SupplementalMaterials.docx 